\title{
Hypertension and abnormal fat distribution but not insulin resistance in mice with P465L PPAR $\gamma$
}

\author{
Yau-Sheng Tsai, ${ }^{1}$ Hyo-Jeong Kim, ${ }^{2}$ Nobuyuki Takahashi, ${ }^{1}$ Hyung-Suk Kim, ${ }^{1}$ \\ John R. Hagaman, ${ }^{1}$ Jason K. Kim, ${ }^{2}$ and Nobuyo Maeda ${ }^{1}$ \\ ${ }^{1}$ Department of Pathology and Laboratory Medicine, University of North Carolina, Chapel Hill, North Carolina, USA. \\ 2Department of Internal Medicine, Section of Endocrinology and Metabolism, Yale University School of Medicine, New Haven, Connecticut, USA.
}

\begin{abstract}
Peroxisome proliferator-activated receptor $\gamma(\operatorname{PPAR} \gamma)$, the molecular target of a class of insulin sensitizers, regulates adipocyte differentiation and lipid metabolism. A dominant negative $P 467 \mathrm{~L}$ mutation in the ligand-binding domain of PPAR $\gamma$ in humans is associated with severe insulin resistance and hypertension. Homozygous mice with the equivalent P465 L mutation die in utero. Heterozygous mice grow normally and have normal total adipose tissue weight. However, they have reduced interscapular brown adipose tissue and intra-abdominal fat mass, and increased extra-abdominal subcutaneous fat, compared with wild-type mice. They have normal plasma glucose levels and insulin sensitivity, and increased glucose tolerance. However, during high-fat feeding, their plasma insulin levels are mildly elevated in association with a significant increase in pancreatic islet mass. They are hypertensive, and expression of the angiotensinogen gene is increased in their subcutaneous adipose tissues. The effects of $\mathrm{P} 465 \mathrm{~L}$ on blood pressure, fat distribution, and insulin sensitivity are the same in both male and female mice regardless of diet and age. Thus the P465L mutation alone is sufficient to cause abnormal fat distribution and hypertension but not insulin resistance in mice. These results provide genetic evidence for a critical role for PPAR $\gamma$ in blood pressure regulation that is not dependent on altered insulin sensitivity.
\end{abstract}

\section{Introduction}

Insulin resistance is the chief abnormality present in metabolic syndrome, a complex physiological condition in which obesity, hypertension, dyslipidemia, and diabetes mellitus coexist in a single individual (1). Thiazolidinediones (TZDs), a class of insulin sensitizers, mediate antidiabetic actions by interacting with the nuclear receptor peroxisome proliferator-activated receptor $\gamma$ $(\operatorname{PPAR} \gamma)(2)$. PPAR $\gamma$ is expressed mainly in adipose tissue and is also detectable in the liver, kidney, intestine, and skeletal muscle. PPAR $\gamma$ heterodimerizes with retinoid $X$ receptor (RXR) and regulates the transcription of target genes through binding to specific peroxisome proliferator response elements. The majority of known target genes are involved in glucose and lipid metabolism. To date, the endogenous PPAR $\gamma$ ligands include various fatty acids and their metabolites (2). These findings indicate that PPAR $\gamma$ functions as a lipid sensor, regulating energy homeostasis. The importance of PPAR $\gamma$ in adipogenesis has been demonstrated by its early expression in preadipocyte differentiation (3). Lack of PPAR $\gamma$ causes embryonic lethality in mice $(4,5)$, and PPAR $\gamma$-null embryos rescued by tetraploid aggregation have no discernible

Nonstandard abbreviations used: adipocyte fatty acid-binding protein (aP2); angiotensin I (AngI); angiotensin II (ATII); angiotensin II type I receptor (AT1R); angiotensin II type II receptor (AT2R); angiotensinogen (AGT); brown adipose tissue (BAT); glucose transporter 4 (Glut4); hepatic glucose production (HGP); heterozygous for PPAR $\gamma$ deficiency (Pparg $\left.{ }^{+/-}\right)$; intraperitoneal glucose tolerance test (IPGTT); intraperitoneal insulin tolerance test (IPITT); lipoprotein lipase (LPL); peroxisome proliferator-activated receptor $\gamma$ (PPAR $\gamma$ ); phosphoenolpyruvate carboxykinase (PEPCK); renin-angiotensin system (RAS); retinoid X receptor (RXR); thiazolidinedione (TZD); uncoupling protein 1 (UCP1).

Conflict of interest: The authors have declared that no conflict of interest exists.

Citation for this article: J. Clin. Invest. 114:240-249 (2004)

doi:10.1172/JCI200420964 white adipose tissue (4). Furthermore, loss-of-function mutations in the ligand-binding domain of PPAR $\gamma$ are associated with lipodystrophy in humans (6-8).

Several lines of evidence support the idea of involvement of PPAR $\gamma$ in the regulation of glucose homeostasis and insulin sensitivity (2). Although activation of PPAR $\gamma$ by TZD is beneficial in improving insulin sensitivity, mice heterozygous for PPAR $\gamma$ deficiency (Pparg ${ }^{+/-}$mice) with reduced PPAR $\gamma$ activity are protected from the insulin resistance induced by a high-fat diet (5). Similarly, in humans, the lower PPAR $\gamma$ activity linked to the PPAR $\gamma$ polymorphism P12A is associated with enhanced insulin sensitivity and reduced body weight (9). Higher than normal PPAR $\gamma$ activity with the P115Q mutation is observed in severely obese subjects with mildly decreased insulin sensitivity (10). In contrast, two dominant negative mutations, P467L and V290M, with reduced PPAR $\gamma$ activity were found among patients with severe insulin resistance and diabetes (11). Thus, it is not clear how aberrant PPAR $\gamma$ signaling relates to insulin resistance and metabolic syndrome.

Hypertension is frequently associated with obesity (12) and modestly with insulin resistance (13), but the genetic and physiological mechanisms underlying these associations are not fully elucidated. Hyperinsulinemia, a consequence of insulin resistance, can affect blood pressure (BP) through increased sodium reabsorption, proliferation of vascular smooth muscle cells, and activation of sympathetic outflow (14). In addition, several components of the renin-angiotensin system (RAS), a key determinant of BP, are expressed in adipose tissue (15), providing a potential link between adipose tissue and hypertension. In rodent models, obese Zucker rats have increased angiotensinogen (AGT) expression and secretion from adipose tissue (16). Transgenic mice overexpressing AGT in adipose tissue have higher BP and increased fat mass (17). These 
A

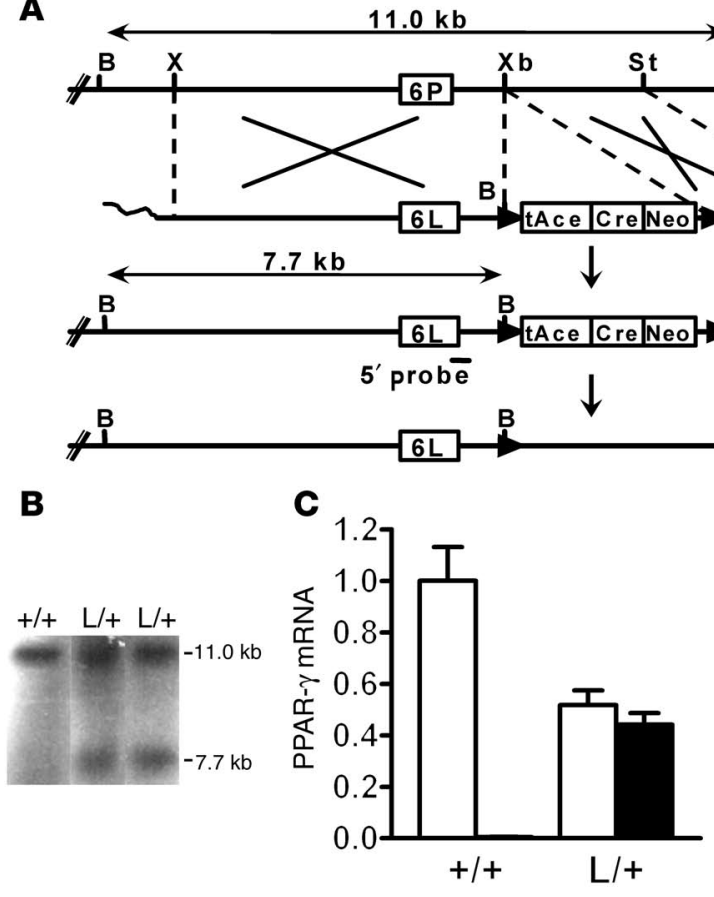

Figure 1

Generation of mice with the PPAR $\gamma$ P465L mutation. (A) Crossovers (indicated by the large X marks) between the wild-type mouse Pparg locus with Pro465 in exon 6 (6P) (top diagram) and targeting construct with Leu465 (6L) (second diagram) resulted in the targeted allele in ES cells (third diagram). The ACN (Cre-Neo) cassette, flanked by loxP sequences, was excised out of the mutant allele upon germline transmission (bottom diagram). B, X, Xb, and St indicate the BamHI, Xhol, Xbal, and Stul restriction enzyme sites, respectively. tAce, testis-specific Ace promoter; TK, thymidine kinase. (B) Southern blot analysis of genomic DNA. The targeted allele was identified by a $5^{\prime}$ probe that hybridizes to an 11-kb fragment in wild-type (+/+) DNA and to a 7.7-kb fragment in heterozygous DNA that includes the P465L mutation (L/+). (C) PPAR $\gamma$ mRNA of the wild-type (white bars) and mutant allele (black bar) in gonadal adipose tissue from wild-type and $P_{p a r g}{ }^{P 465 /+}$ mice $(n=8$ each). The PPAR $\gamma$ mRNA amount is expressed relative to that of wild-type allele in wild-type mice. (D) Rosiglitazone-induced PEPCK expression in gonadal adipose tissue explants. Tissues isolated from four wild-type (open squares) and four PpargP465L/+ (filled squares) mice were incubated in cultured media containing various concentrations of PPAR $\gamma$ agonist rosiglitazone as indicated. The levels of PEPCK mRNA are relative to the wildtype basal level. ${ }^{*} P<0.01$ and ${ }^{* *} P<0.005$ compared with the respective basal levels.

findings support the idea of a potential role for the adipose RAS in the development of obesity and obesity-related hypertension.

TZDs are reported to decrease BP in hypertensive rat models (18), as well as in human patients (19), and are thought to directly regulate BP through PPAR $\gamma$ in vascular smooth muscle cells (20) and endothelial cells $(21,22)$. Patients with the dominant negative mutations P467L and V290M in PPAR $\gamma$ were noted to have early-onset hypertension (11). However, the mechanism by which these dominant negative mutations lead to insulin resistance and hypertension in vivo is not known, and whether the elevated BP is dependent on or independent of altered insulin sensitivity is not clear.

To determine whether the P467L mutation directly causes insulin resistance and hypertension, we have generated mice in which the proline at position 465 of the mouse PPAR $\gamma$ protein (equivalent to residue 467 in the human protein) is substituted with leucine through gene targeting. Homozygous Pparg ${ }^{\text {P465L/P465L }}$ mice die in utero. Heterozygous Pparg ${ }^{\mathrm{P} 465 \mathrm{~L} / \mathrm{t}}$ mice exhibit abnormalities seen in the human $\mathrm{P} 467 \mathrm{~L}$ patients, including elevated BP
(11) and abnormal fat distribution (6), but unlike humans, they maintain normal insulin sensitivity.

\section{Results}

Mice with the PPAR P465L mutation. We mutated codon 465 of the mouse Pparg gene (equivalent to codon 467 in human PPARG) from CCC (proline) to CTG (leucine) via gene targeting in mouse ES cells, and generated mice carrying the P465L substitution (Figure 1A). Southern blots (Figure 1B) and nucleotide sequence analyses (data not shown) confirmed the mutation in the Pparg gene. Wildtype and heterozygous Pparg ${ }^{P 465 L /+}$ littermates were born at the expected mendelian ratio (34:61) from mating of heterozygous pairs, but no homozygous Pparg ${ }^{\text {P465L/P465L mice }}$ were born. Thus, homozygosity for the P465L mutation is lethal in utero, establishing that the mutant protein is effectively nonfunctional. For the following studies, we used $F_{1}$ offspring derived from crosses between 129/SvEv heterozygous Pparg ${ }^{P 465 L /+}$ mice and C57BL/ 6 wild-type mice.

Precision quantitative RT-PCR revealed that expression of the wildtype allele in adipose tissues of Pparg $^{\text {P465L/+ }}$ mice was, as expected, about $50 \%$ of that in wild-type littermates (Figure 1C). The mutant P465L transcripts in Pparg ${ }^{P 465 L /+}$ mice were present at the same level as the wild-type transcripts, showing that the mutant allele is transcribed normally. Total amounts of PPAR $\gamma$ transcripts were not different between $\mathrm{Pparg}^{\mathrm{P465L} /{ }^{+}}$and wild-type mice. Similarly, there was no compensatory alteration in the gene expression of RXR $\alpha, \operatorname{PPAR} \alpha$, or PPAR $\delta$ (data not shown).

Cultured gonadal adipose tissue isolated from wild-type mice responded to rosiglitazone by increasing expression of the PPAR $\gamma$ target gene encoding phosphoenolpyruvate carboxykinase (PEPCK) in a dose-dependent manner (Figure 1D). In contrast, activation of PEPCK expression was markedly impaired in cultured adipose tissue from $P$ parg ${ }^{P 465 L /+}$ mice (Figure 1D), indicating that rosiglitazone-dependent activation of wild-type PPAR $\gamma$ is dominantly suppressed by the $\mathrm{P} 465 \mathrm{~L}$ protein in vivo.

Abnormal body fat distribution in Pparg ${ }^{P 465 L /+}$ mice. Pparg ${ }^{P 465 L /+}$ mice developed normally and appeared healthy. Their food and water intake, urine output, rectal temperature, and hematological parameters, and the macroscopic and microscopic features of their major organs were normal (data not shown). Fasting plasma lipids, including total cholesterol, triglyceride, and FFA, were not altered in Pparg ${ }^{P 465 L /+}$ mice (Table 1). The growth curves of both male and female Pparg ${ }^{P 465 L /+}$ mice were the same as those of wildtype mice fed regular chow or a high-fat diet (Figure 2A). Organ 


\section{Table 1}

Metabolic parameters in PpargP465L/+ (L/+) and WT male mice

\begin{tabular}{|c|c|c|c|c|c|c|c|c|c|c|}
\hline \multirow{3}{*}{$\begin{array}{l}\text { Age } \\
\text { Diet } \\
\text { Genotype }\end{array}$} & \multicolumn{6}{|c|}{4 months } & \multicolumn{4}{|c|}{9 months } \\
\hline & \multicolumn{2}{|c|}{ Regular chow } & \multicolumn{2}{|c|}{ High fat ${ }^{A}$} & \multicolumn{2}{|c|}{ High salt ${ }^{A}$} & \multicolumn{2}{|c|}{ Regular chow } & \multicolumn{2}{|c|}{ High fat ${ }^{B}$} \\
\hline & $\mathrm{L} /+$ & WT & $\mathrm{L} /+$ & WT & $\mathrm{L} /+$ & WT & $\mathrm{L} /+$ & WT & $\mathrm{L} /+$ & WT \\
\hline BW $(g)$ & $30.6 \pm 1.6$ & $31.8 \pm 1.3$ & $36.9 \pm 0.7$ & $35.2 \pm 1.0$ & $27.1 \pm 0.8$ & $29.1 \pm 0.9$ & $37.2 \pm 2.0$ & $40.9 \pm 1.7$ & $48.7 \pm 1.7$ & $47.9 \pm 2.5$ \\
\hline Gonadal (\%BW) & $0.8 \pm 0.2$ & $1.2 \pm 0.2$ & $3.8 \pm 0.4^{C}$ & $4.9 \pm 0.3$ & n.d. & n.d. & n.d. & n.d. & $6.4 \pm 0.5$ & $6.9 \pm 0.5$ \\
\hline Inguinal (\%BW) & $0.8 \pm 0.1$ & $0.6 \pm 0.1$ & $3.8 \pm 0.4^{\mathrm{D}}$ & $2.4 \pm 0.2$ & n.d. & n.d. & n.d. & n.d. & $4.2 \pm 0.2^{D}$ & $3.3 \pm 0.2$ \\
\hline $\mathrm{G} / \mathrm{I}$ fat ratio & $1.0 \pm 0.0^{\mathrm{E}}$ & $2.0 \pm 0.1$ & $1.0 \pm 0.1^{\mathrm{E}}$ & $2.1 \pm 0.1$ & n.d. & n.d. & n.d. & n.d. & $1.5 \pm 0.1^{\mathrm{D}}$ & $2.1 \pm 0.1$ \\
\hline Glucose (mg/dl) & $142 \pm 6$ & $141 \pm 7$ & $190 \pm 15$ & $214 \pm 20$ & $215 \pm 17$ & $221 \pm 8$ & $198 \pm 8$ & $179 \pm 9$ & $213 \pm 8$ & $241 \pm 17$ \\
\hline Insulin (ng/ml) & $0.6 \pm 0.1$ & $0.4 \pm 0.2$ & $3.0 \pm 0.8$ & $2.0 \pm 0.3$ & $0.8 \pm 0.3$ & $0.5 \pm 0.3$ & $1.4 \pm 0.4$ & $1.0 \pm 0.3$ & $2.0 \pm 0.4$ & $1.5 \pm 0.4$ \\
\hline IR index $(\%)$ & $6.8 \pm 0.9$ & $8.0 \pm 1.3$ & $25.3 \pm 3.1$ & $21.4 \pm 2.6$ & $7.3 \pm 1.1$ & $9.2 \pm 3.2$ & $17.0 \pm 5.5$ & $13.5 \pm 3.1$ & $23.0 \pm 2.6$ & $19.1 \pm 3.1$ \\
\hline $\mathrm{TG}(\mathrm{mg} / \mathrm{dl})$ & $28 \pm 3$ & $29 \pm 3$ & $58 \pm 6$ & $62 \pm 7$ & $53 \pm 4$ & $48 \pm 4$ & $71 \pm 7$ & $54 \pm 6$ & $60 \pm 8$ & $73 \pm 8$ \\
\hline Chol (mg/dl) & $85 \pm 3$ & $79 \pm 3$ & $114 \pm 7$ & $124 \pm 6$ & $113 \pm 4$ & $115 \pm 4$ & $107 \pm 4$ & $96 \pm 4$ & $136 \pm 5$ & $146 \pm 7$ \\
\hline $\mathrm{FFA}(\mathrm{mmol} / \mathrm{l})$ & $0.9 \pm 0.1$ & $1.0 \pm 0.1$ & $1.4 \pm 0.1$ & $1.2 \pm 0.0$ & $0.9 \pm 0.1$ & $0.8 \pm 0.1$ & $0.8 \pm 0.1$ & $0.8 \pm 0.1$ & $1.4 \pm 0.0$ & $1.3 \pm 0.2$ \\
\hline $\mathrm{BP}(\mathrm{mmHg})$ & $120 \pm 3^{c}$ & $111 \pm 3$ & $118 \pm 3$ & $113 \pm 3$ & $120 \pm 3^{c}$ & $111 \pm 3$ & $120 \pm 3^{c}$ & $112 \pm 2$ & $122 \pm 4$ & $109 \pm 5$ \\
\hline PR (beats/min) & $600 \pm 6$ & $583 \pm 12$ & $652 \pm 10$ & $638 \pm 6$ & $623 \pm 8$ & $631 \pm 8$ & $674 \pm 8$ & $670 \pm 9$ & $672 \pm 15$ & $684 \pm 7$ \\
\hline
\end{tabular}

AMice were fed a high-fat or high-salt diet for 1 month. ${ }^{\mathrm{B} M i c e}$ were fed a high-fat for 6 months. ${ }^{\mathrm{C} P}<0.05$, ${ }^{\mathrm{D} P}<0.01$, and $\mathrm{E} P<0.001$ between $P p a r g P 465 L /+$ and wild-type littermates within each group. BW, body weight; G/I, gonadal/inguinal; IR, insulin resistance; TG, triglyceride; Chol, cholesterol; PR, pulse rate; n.d., not determined.

and total weights of abdominal fat pads (gonadal, mesenteric, retroperitoneal, and inguinal, normalized by body weight) were not significantly different between mice of the two genotypes.

Despite the similar total body fat in $\mathrm{Pparg}^{\mathrm{P4} 65 \mathrm{~L} /{ }^{+}}$and wild-type mice, we found significant differences in their fat distribution (Figure 2B). The interscapular brown adipose tissue (BAT) weight relative to body weight of $\mathrm{Pparg}^{\mathrm{P} 465 \mathrm{~L} /+}$ mice was about $80 \%$ that of wild-type mice $(P<0.02)$, and the gonadal fat mass in $P$ parg ${ }^{P 465 L /+}$ mice was about $70 \%(P<0.004)$. In contrast, the weight of the inguinal fat pad in $P$ parg ${ }^{P 465 L /+}$ mice was increased to about $140 \%$ that of wild-type mice $(P<0.01)$. The pectoral subcutaneous fat pad in $\mathrm{Pparg} \mathrm{P}^{\mathrm{P} 65 \mathrm{~L} / \mathrm{+}}$ mice was similarly increased $(P<0.02)$. Consequently, the ratio of intra-abdominal fat weight to extra-abdominal fat weight (the sum of mesenteric, gonadal, and retroperitoneal fat depots versus inguinal fat) in $P_{p a r g}{ }^{P 465 L /+}$ mice was substantially reduced to $63 \%$ that of wild-type mice $(P<0.0001)$. A high-fat diet led to fat deposition in both mutant and wild-type mice and increased this difference in fat distribution (47\% of wild-type; $P<0.0001$ ).

Microscopically, the cellularity of adipose tissues in wild-type mice was heterogeneous. In contrast, the adipocytes in $P$ parg ${ }^{P 465 L /+}$ mice were relatively uniform and the number of small adipocytes was reduced (Figure 2C). The size distributions of adipocytes in the gonadal and inguinal fat from $\mathrm{Pparg}^{\mathrm{P465L} /+}$ mice showed significant shifts toward larger cells compared with the distributions from wild-type mice (Figure 2D). Retroperitoneal, mesenteric, and pectoral fat showed a similar but less marked trend. These results suggest that the increase in inguinal fat mass of Pparg ${ }^{P 465 L /+}$ mice is mainly due to an increase in triacylglycerol storage, while the decrease in gonadal fat mass is due to a decrease in cell number. Both the gonadal and inguinal fat pads of Pparg ${ }^{P 465 L /+}$ mice showed a decrease in the number of multilocular adipocytes (Figure $2 \mathrm{C}$ ). Consistent with this, we found significant reductions in mRNA for the brown fat-specific protein uncoupling protein 1 (UCP1) in gonadal and inguinal adipose tissues to $37 \%(P<0.02)$ and $29 \%(P<0.03)$ that of wild-type mice, respectively (Figure $2 \mathrm{E})$. The amount of UCP1 mRNA in interscapular BAT did not differ between $\mathrm{Pparg}^{\mathrm{P465L/+}}$ and wild-type mice (Figure 2E).
To examine the in vivo effects of the $\mathrm{P} 465 \mathrm{~L}$ mutation on the expression of PPAR $\gamma$ target genes, we measured their steadystate mRNA levels in adipose tissues. Despite the impaired PEPCK activation in culture described above, expression of the gene encoding PEPCK in both gonadal and inguinal adipose tissues of $\mathrm{Pparg}^{\mathrm{P465L} /{ }^{+}}$mice was indistinguishable from that of wildtype mice (Figure 2F). In contrast, mRNA levels for adipocyte fatty acid-binding protein (aP2; reduced to $0.58 \times$ wild-type levels, $P<0.002$ ) and glucose transporter 4 (Glut4; reduced to $0.64 \times$ wild-type levels, $P<0.05)$ were significantly decreased in gonadal but not inguinal adipose tissue in $\mathrm{Pparg}^{\mathrm{P} 465 \mathrm{~L} /{ }^{+}}$mice. Conversely, expression of the gene encoding lipoprotein lipase (LPL) was not affected in gonadal adipose tissue but was significantly increased in inguinal adipose tissue of $\mathrm{Pparg}^{\mathrm{P465L/+}}$ mice (increased to $3.48 \times$ wild-type levels, $P<0.05$ ). Thus, the presence of the P465L mutation does not lead to a uniform change, but exerts adipose depot-specific effects on the basal expression of PPAR $\gamma$ target genes in vivo.

These results demonstrate that the P465L mutation in PPAR $\gamma$ causes abnormal fat distribution, leading to a preferential deposition of fat in subcutaneous fat pads rather than in intra-abdominal fat pads, and reduces the number of small adipocytes, including mutilocular adipocytes, in these white fat depots.

Normal insulin sensitivity in Pparg ${ }^{P 465 L /+}$ mice. The human $\mathrm{P} 467 \mathrm{~L}$ mutation is associated with severe insulin resistance, hyperinsulinemia, and diabetes. However, the plasma glucose concentrations in $\mathrm{Pparg}^{\mathrm{P} 465 \mathrm{~L} /+}$ mice after fasting were normal compared with those of wild-type mice (Table 1). Their plasma insulin levels were slightly but not significantly higher than those of wild-type mice. Feeding the mice a diet high in fat for 4 weeks substantially increased plasma glucose and insulin levels in both $P_{p a r g}{ }^{P 465 L /+}$ and wild-type mice compared with feeding the mice regular chow. The Pparg ${ }^{P 465 L /+}$ mice fed the high-fat diet had slightly lower glucose and higher insulin levels than did their wild-type littermates fed a similar diet (Table 1). These differences were not statistically significant, however.

To assess the dynamic response of $\mathrm{Pparg}^{\mathrm{P465L/+}}$ mice to increased glucose concentrations, we performed the intraperitoneal glucose 
A

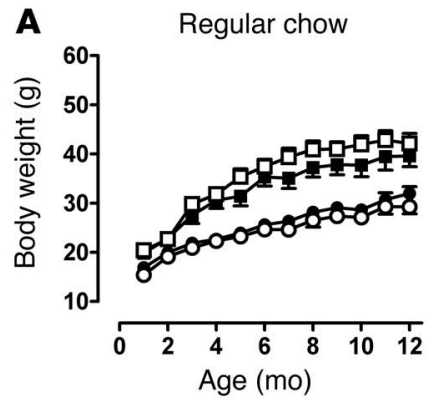

c

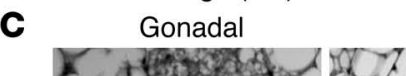
Inguinal
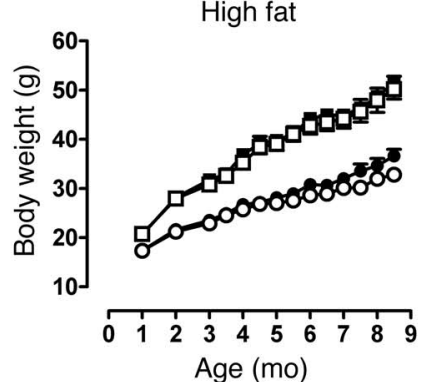

Age (mo)
BAT

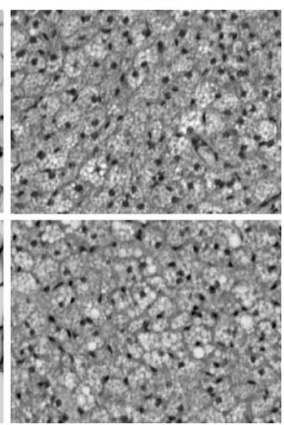

D

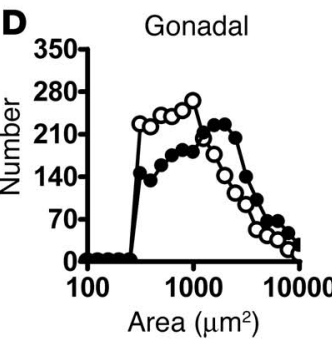

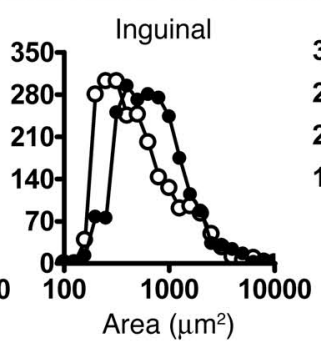

Retroperitoneal

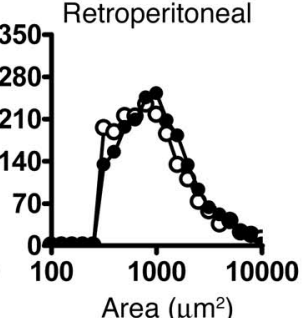

B

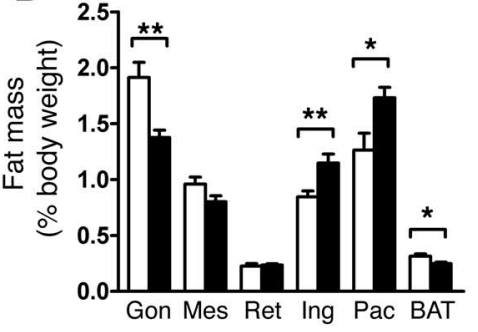

E

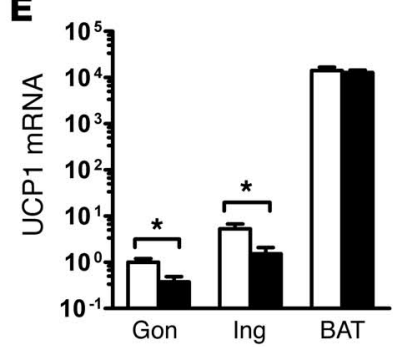

$\mathbf{F}$

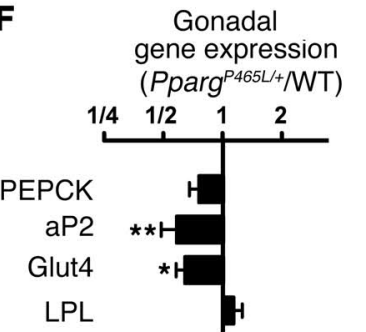

Inguinal gene expression

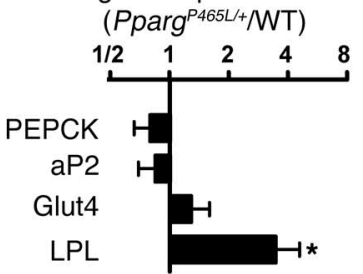

Figure 2

Altered adipose tissue distribution in Pparg ${ }^{P 465 L /+}$ mice. (A) Body weights of male mice (open squares, wild-type, $n=9-10$; filled squares, Pparg ${ }^{P 465 L /+}, n=9-10$ ) and female mice (open circles, wild-type, $n=7-9$; filled circles, Pparg ${ }^{P 465 L /+}, n=8$ ) fed regular chow (left panel) or a high-fat diet (right panel). (B) Adipose tissue mass in 10-week-old female wild-type (white bars) and PpargP465L+ (black bars) mice fed regular chow. Data are expressed as percent body weights. Gon, Mes, Ret, Ing, and Pec represent gonadal, mesenteric, retroperitoneal, inguinal, and pectoral white adipose tissue, respectively; BAT indicates interscapular BAT. ${ }^{\star} P<0.05$ and ${ }^{\star \star} P<0.01$, compared with wild-type littermates. (C) Morphology of gonadal and inguinal white adipose and brown adipose tissues from 10-week-old female mice fed regular chow. +/+, wild-type; L/+, Pparg ${ }^{P 465 L /+}$. (D) Distribution of cell size in gonadal (left panel), inguinal (middle panel), and retroperitoneal (right panel) adipose tissues. Open circles, wild-type; filled circles, $P$ parg ${ }^{P 465 L /+}$. (E) UCP1 mRNA relative to the level of gonadal adipose tissue from wild-type mice. ${ }^{\star} P<0.05$ between $P p a r g P 465 L /+$ and wild-type littermates ( $n=16$ each). The $y$ axis is in a log scale. (F) Relative ratio of mRNA for PPAR target genes in gonadal and inguinal adipose tissues of $P$ parg ${ }^{P 465 L /+}$ mice to those of wild-type mice $\left(n=16\right.$ each). ${ }^{*} P<0.05$ and ${ }^{\star *} P<0.005$ between $P$ parg ${ }^{P 465 L /+}$ and wild-type littermates.

tolerance test (IPGTT). Pparg ${ }^{P 465 L /+}$ mice cleared glucose faster than their wild-type littermates did, regardless of diet, indicating improved glucose tolerance in the mutant mice (Figure $3 \mathrm{~A}$ ). This improved glucose tolerance was accompanied by significantly elevated plasma insulin levels ( $P<0.05$ at 15 minutes) during the IPGTTs of PpargP ${ }^{P 65 L /+}$ mice fed a high-fat diet but not of mice fed regular chow. The insulin resistance index of $P$ parg ${ }^{P 465 L /+}$ mice calculated from the IPGTT was slightly lower in the group fed regular chow but higher in the group fed a high-fat diet than that of wildtype mice (Table 1). However, these differences were not significant. Consistent with these findings, the intraperitoneal insulin tolerance test (IPITT) revealed that the hypoglycemic response to an acute administration of insulin $(0.5 \mathrm{U} / \mathrm{kg})$ was normal in Pparg ${ }^{P 465 L /+}$ mice fed regular chow or a high-fat diet (Figure 3B).

To further examine the insulin sensitivity of $\mathrm{Pparg}^{\mathrm{P465L/+}}$ mice in vivo, we established a 2-hour hyperinsulinemic-euglycemic clamp in conscious mice fed regular chow or a high-fat diet for 3 weeks. The rates of glucose infusion required to maintain the euglycemic clamp were not different for $\mathrm{Pparg}^{\mathrm{P465L/+}}$ mice fed regular chow compared with their wild-type littermates (Figure 3C). Feeding mice a high-fat diet blunted the insulin response during the clamps to the same extent in both groups, as reflected by their significantly lower but equal steady-state glucose infusion rates. Insulin-stimulated whole-body glucose turnover rates were not different in mice of the two genotypes fed regular chow and were similarly reduced after mice were fed a high-fat diet. No genotype effect was observed in insulin-stimulated wholebody glycolysis, glycogen/lipid synthesis (data not shown), and hepatic glucose production (HGP) in basal and clamped states (Figure 3C). These results indicate that the Pparg ${ }^{P 465 L /+}$ mutation does not alter peripheral or hepatic insulin sensitivity and suggest that the increased glucose tolerance of $\mathrm{Pparg}^{\mathrm{P465L/+}}$ mice is 

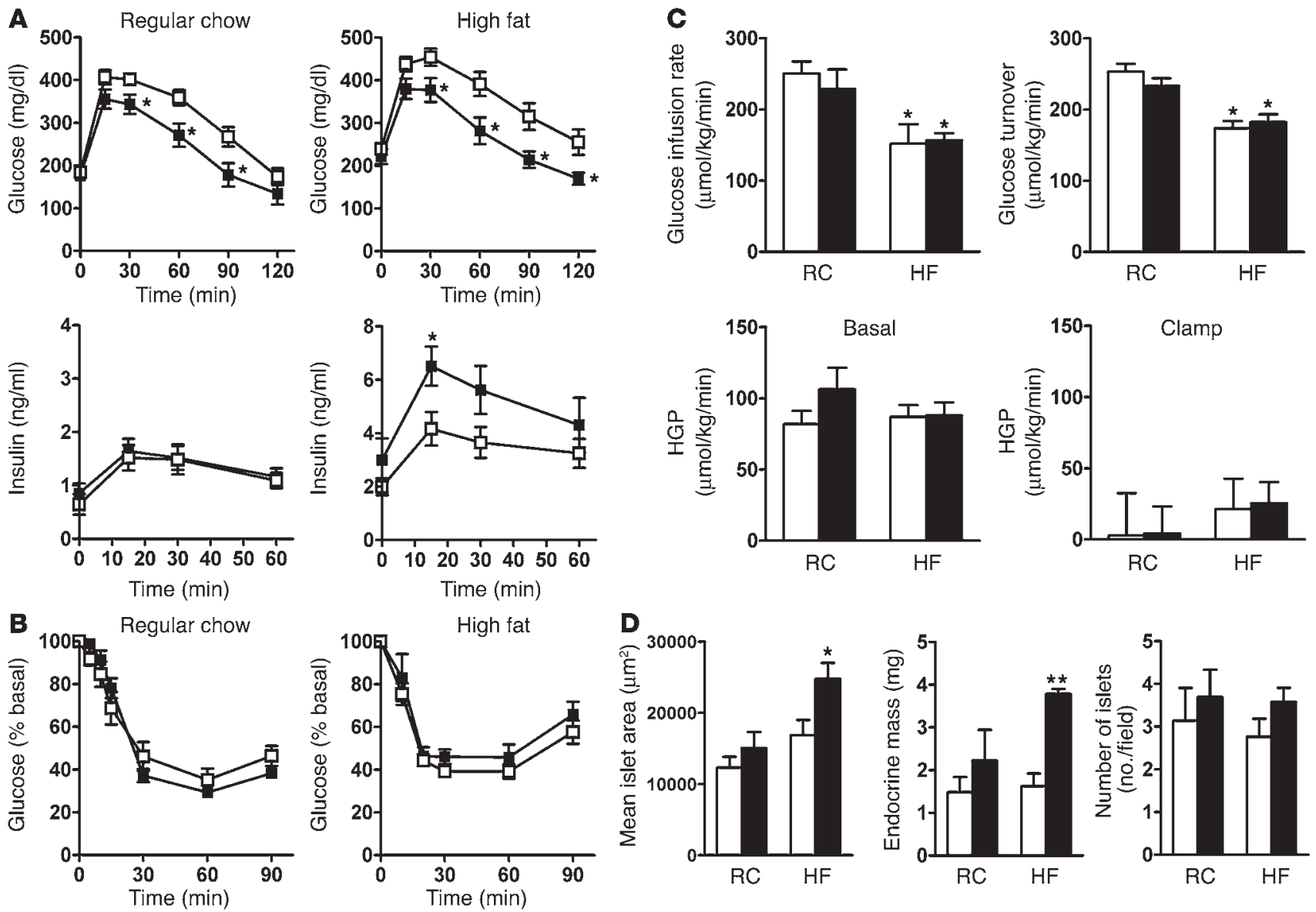

Figure 3

Increased glucose tolerance but normal insulin sensitivity in Pparg ${ }^{P 465 L /+}$ mice. (A) Plasma glucose and insulin levels during the IPGTT in 14to 16-week-old male mice fed regular chow (left panels; $n=15-16$ ) or a high-fat diet (right panels; $n=7-11$ ). Open squares, wild-type; filled squares, $P$ parg ${ }^{P 465 L /+}$. ${ }^{*} P<0.05$ versus wild-type. (B) IPITTs of 14- to 16-week-old female mice fed regular chow (left panel; $n=7-8$ ) or a high-fat diet (right panel; $n=6-7$ ). Open squares, wild-type; filled squares, Pparg ${ }^{P 465 L /+}$. Data are expressed as the percentage of the plasma glucose before insulin injection. (C) Whole-body metabolic parameters during the hyperinsulinemic-euglycemic clamp experiment. Steady-state glucose infusion rates (top left) and insulin-stimulated whole-body glucose turnover rates (top right) were obtained for 10- to 12-week-old male mice fed regular chow $(\mathrm{RC} ; n=5)$ or a high-fat diet (HF; $n=5-8)$. White bars, wild-type; black bars, $P$ parg $465 L /+.{ }^{*} P<0.05$ for diet effect. Basal (bottom left) and clamped (bottom right) rates of HGP in wild-type (white bars) and Pparg $465 \mathrm{~L} /+$ (black bars) mice fed regular chow (RC; $n=5)$ or a high-fat diet (HF; $n=5-8)$. (D) Morphometric analysis of pancreatic islets. Mean islet area (left), pancreatic endocrine mass (middle), and islet number (right) were measured in sections from 14- to 16-week-old male mice fed regular chow $(\mathrm{RC} ; n=4)$ or a high-fat diet $(\mathrm{HF} ; n=4)$. White bars, wild-type; black bars, $P$ parg ${ }^{465 L /+}$. ${ }^{*} P<0.05$ and ${ }^{* *} P<0.001$ compared with wild-type littermates.

due mainly to their elevated plasma insulin rather than to an altered insulin sensitivity.

We next examined pancreatic islet morphology. While there was no change in the weight of pancreas (data not shown), the mean islet area and endocrine mass in 4-month-old Pparg ${ }^{P 465 L /+}$ mice were both slightly increased in the group fed regular chow and were significantly increased in the group fed a high-fat diet (Figure 3D). The number of islets was similar in $\mathrm{Pparg}^{\mathrm{P4}}{ }^{\mathrm{SL} / \mathrm{+}}$ and wild-type mice. Thus, increased endocrine mass and islet size may be responsible for the observed elevation of plasma insulin levels during glucose overload in Pparg ${ }^{P 465 L /+}$ mice.

Elevated BP in Pparg ${ }^{P 465 L /+}$ mice. The P465L mutation in PPAR $\gamma$ caused a significant increase of about $8 \mathrm{mmHg}$ in the BP of both male and female mice, as measured by a tail cuff method $(P<0.01$ for genotype effect but not significant for gender effect by twoway ANOVA; Figure 4A). The pulse rates of Pparg ${ }^{P 465 L /+}$ mice were not different from those of their wild-type littermates (Table 1). Consistent with the tail-cuff measurements, telemetric BP monitoring showed higher than average systolic/diastolic BP during the light cycle in the Pparg ${ }^{P 465 L /+}$ mice $(132 / 94 \mathrm{mmHg}$ versus wild-type $110 / 82 \mathrm{mmHg}$ ) (Figure 4B). BP during the dark cycle was similarly elevated in Pparg ${ }^{P 465 L /+}$ mice $(144 / 103 \mathrm{mmHg}$ versus wild-type $124 / 95 \mathrm{mmHg}$ ) (Figure 4B). Thus, the diurnal rhythm of BP is maintained in $\mathrm{Pparg}^{\mathrm{P4} 65 \mathrm{~L} / \mathrm{+}}$ mice and the elevation of their BP in the active phase is not disproportionate.

Because PPAR $\gamma$ is expressed in the renal medulla (23), we next examined the possibility that $P$ parg ${ }^{P 465 L /+}$ mice may have abnormal salt and water reabsorption in the kidney and may display saltsensitive hypertension. Feeding mice a high-salt diet containing $8 \% \mathrm{NaCl}$ for 4 weeks did not change the BP of either Pparg ${ }^{P 465 L /+}$ or wild-type mice, and the genotype effect on BP was maintained (Figure 4C). We further tested the salt sensitivity of $\mathrm{Pparg}^{P 465 L /+}$ 
A
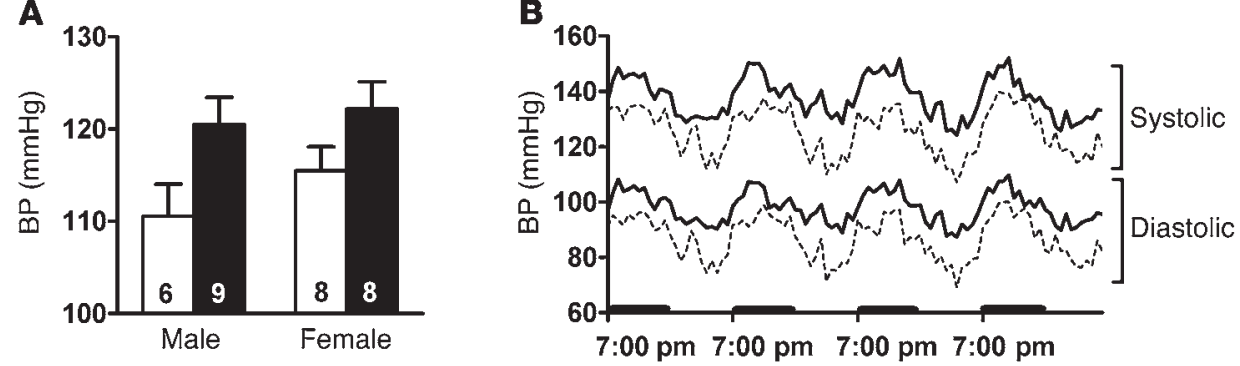

C
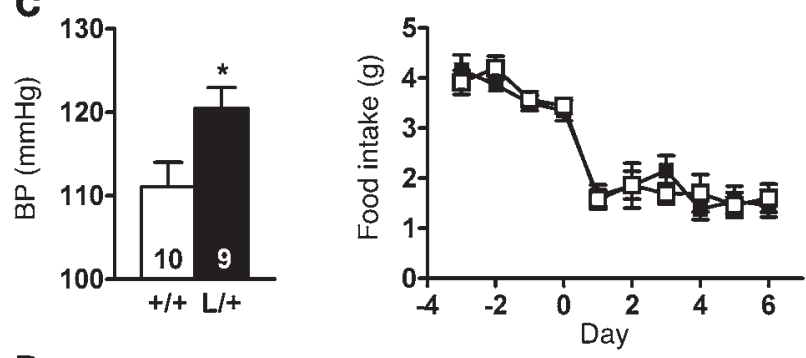

Inguinal adipose

D tissue mRNA $\left(\right.$ Pparg $^{\text {P465L }+/ N T)}$

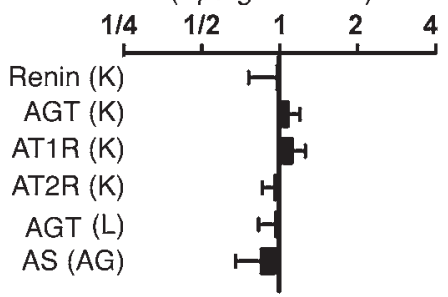

$\left(\right.$ Pparg $^{\text {P465L+/WT) }}$

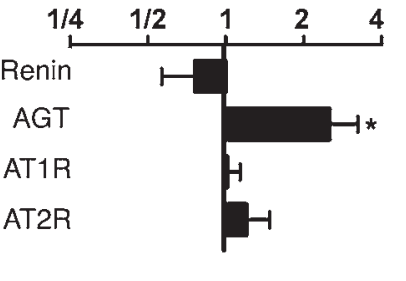

Figure 4

Elevated BP in Pparg ${ }^{P 465 L /+}$ mice. (A) BP of 14- to 16-week-old wild-type (white bars) and Pparg ${ }^{P 465 L /+}$ (black bars) mice by tail-cuff measurement. Numbers of mice are inside bars. $P<0.01$ for genotype effect by ANOVA. (B) Four-day telemetric recordings of systolic and diastolic BP in 24-week-old female mice. Results are expressed as mean of four wild-type (dashed lines) and four Pparg ${ }^{P 465 L /+}$ (solid lines) mice averaged with 12 values each hour. Bolded bars on the $x$ axis represent the dark cycles. (C) Responses to changes in dietary salt intake. BP of 14- to 16-week-old male wild-type (white bar) and Pparg ${ }^{P 465 L /+}$ (black bar) mice fed a high-salt diet for 4 weeks (left panel). Numbers inside bars indicate sample size. ${ }^{\star} P<0.05$. Changes in daily food consumption (middle panel) and urinary sodium excretion (UNaV; right panel) after mice were switched to a high-salt diet at day 0 for 10- to 12-week-old male wild-type (open squares, $n=7$ ) and Pparg ${ }^{P 465 L /+}$ (filled squares, $n=6$ ) mice. (D) Relative ratio of the expression of RAS genes in Pparg ${ }^{P 465 /+}$ mice to those in wild-type mice ( $n=16$ each). Left panel, organs of major RAS expression: K, kidney; L, liver; AG, adrenal gland. Middle panel, inguinal adipose tissue. Right panel, gonadal adipose tissue. ${ }^{*} P<0.05$ and ${ }^{* *} P<0.005$ between $P$ parg ${ }^{P 465 L /+}$ and wild-type mice.

mice during the immediate adaptation to an increased dietary salt load before homeostatic adjustments take place. Changes in daily food consumption were not different, indicating mice had the same salt intake. Urinary sodium excretion was increased considerably on initiation of high-salt feeding. However, the daily sodium excretion as well as 4-day cumulative sodium excretion after initiation of the high-salt diet was not different between mice of the two genotypes. Thus $P$ parg ${ }^{P 465 L /+}$ mice have normal salt and water handling in the kidney and the elevation of BP in Pparg ${ }^{P 465 L /+}$ mice is not salt sensitive.

Because the RAS plays a crucial role in the maintenance of BP, we measured the steady-state mRNA levels of genes of the RAS. The mRNA levels for AGT, renin, and aldosterone synthase (AS) in the liver, kidney, and adrenal gland, the major sites of their expression, respectively, were not affected by the presence of the P465L

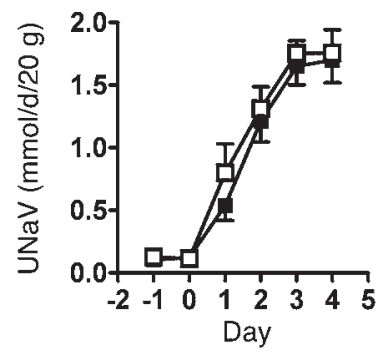

Gonadal adipose tissue mRNA (Pparg $\left.{ }^{\mathrm{P} 65 \mathrm{~L} /}+\mathrm{NT}\right)$

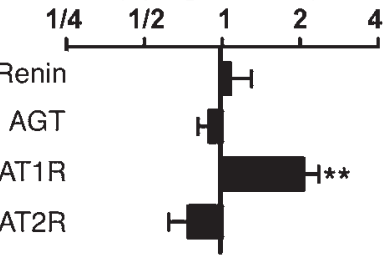

mutation (Figure 4D). PPAR $\gamma$ activators have been shown to transcriptionally suppress the angiotensin II type I receptor (AT1R) (24), but we found that the AT1R mRNA levels in kidney were not different in $\mathrm{Pparg}^{\mathrm{P} 465 \mathrm{~L} /+}$ versus wild-type mice. Consistent with the mRNA levels, plasma renin concentration (42 $\pm 13 \mathrm{ng}$ of angiotensin I/ml/ $\mathrm{h}$ in $\mathrm{Pparg}^{\mathrm{P} 465 \mathrm{~L} /+}$ versus $44 \pm 14$ in wild-type; $P=0.89$ ), plasma AGT levels (equivalent to $622 \pm 7 \mathrm{ng}$ of angiotensin I/ml in Pparg ${ }^{P 465 L /+}$ versus $608 \pm 20$ in wild-type; $P=0.50$ ), and plasma aldosterone (426 $\pm 73 \mathrm{pg} / \mathrm{ml}$ in Pparg $^{\text {P465L/+ }}$ versus $408 \pm 79$ in wild-type; $P=0.87)$ concentrations were not different between mice of the two genotypes. Thus, the systemic RAS does not appear to be altered in P465L PPAR $\gamma$-mediated hypertension.

Adipose tissue also produces and secretes vasoactive precursor substances including AGT, which may contribute to the development of hypertension (17). Expression of the gene encoding for renin in both gonadal and inguinal adipose tissues was low and was not significantly different in mice of the two genotypes (Figure 4D). The mRNA levels for angiotensin II type II receptor (AT2R), which is reported to antagonize AT1R by inducing vasodilation and inhibiting cell growth and proliferation (25), were not different in mice of the two genotypes. In contrast, the level of AGT mRNA in the inguinal fat of $P$ parg ${ }^{P 465 L /+}$ mice was 2.5 times that of wild-type mice $(P<0.05)$. The level of AGT mRNA in the gonadal fat of $P$ parg ${ }^{P 465 L /+}$ mice was not different from that of wild-type mice. Conversely, a significant increase in the AT1R gene expression was observed in the gonadal fat $(2.1 \times$ wild type, $P<0.005)$ but not in the inguinal fat of $P p a r g^{P 465 L /+}$ mice. Thus the genes of the RAS are altered in adipose tissues of $P$ parg ${ }^{P 465 L /+}$ mice in a depot-specific fashion. These data suggest that the elevated BP of Pparg ${ }^{P 465 L /+}$ mice could be affected by changes in the local RAS of adipose tissues.

Effects of age, diet, and gender. Diets containing high fat as well as aging are known to affect weight gain, fat deposition, and insulin sensitivity, which could ultimately affect BP. We therefore examined the phenotypes of mice at 9 months of age that were either maintained on regular chow or fed a high-fat diet for 22 weeks (Table 1). We found significant age and diet effects on body weight, fat mass, 


\section{Table 2}

Phenotypes of humans heterozygous for P467L PPAR $\gamma$, mice heterozygous for P465L PPAR $\gamma$, and mice heterozygous for PPAR $\gamma$ knockout

\begin{tabular}{lccc} 
& Humans & \multicolumn{2}{c}{ Mice } \\
Mutation & P467L/+ & P465L/+ & $+/-$ \\
Body weight & $\leftrightarrow$ & $\leftrightarrow$ & $\downarrow$ \\
Body temperature & $\leftrightarrow$ & $\leftrightarrow$ & $\uparrow$ \\
Blood pressure & $\uparrow$ & $\leftrightarrow$ & $\leftrightarrow$ \\
Insulin sensitivity & $\downarrow$ & Mildly $\uparrow$ & $\downarrow$ \\
Plasma insulin & $\uparrow$ & Tolerant & Tolerant \\
GTT & Intolerant & $\leftrightarrow$ & Sensitive \\
ITT & Resistant & $\leftrightarrow$ & Sensitive \\
Euglycemic clamp & Resistant & Redistribution & General \\
Fat mass alteration & Partial & & reduction \\
& reduction & $\downarrow$ & $\downarrow$ \\
Intra-abdominal fat & $\leftrightarrow$ & $\uparrow$ & $\downarrow$ \\
Sub. abdominal fat & $\leftrightarrow$ & n.d. & n.d. \\
Sub. peripheral fat & $\downarrow$ & No & No \\
Dyslipidemia & Yes & $\leftrightarrow$ & $\leftrightarrow$ \\
Plasma cholesterol & $\leftrightarrow$ & $\leftrightarrow$ & $\leftrightarrow$ \\
HDL cholesterol & $\downarrow$ & $\leftrightarrow$ & $\leftrightarrow$ \\
Plasma triglyceride & $\uparrow$ & & $(5,26,27)$ \\
References & $(6,11)$ & & \\
\hline$\uparrow$, inceas & & & $\leftrightarrow$
\end{tabular}

$\uparrow$, increase; $\downarrow$, decrease; $\leftrightarrow$, not different compared with control population or wild-type mice. Sub., subcutaneous; n.d., not determined.

plasma glucose, and insulin, and insulin resistance index, but the changes induced by diet and age were similar in both Pparg ${ }^{P 465 L /+}$ and wild-type mice. Neither age nor diet influenced BP. Except for the hyperinsulinemic clamp experiments, which were done only in male mice, we studied both male and female mice. The P465L effects on BP, fat distribution, and insulin sensitivity were the same in both male and female mice without any interaction between gender and genotype by ANOVA.

\section{Discussion}

To establish a causative link between the PPAR $\gamma$ P467L mutation and the observed phenotypes in humans, we introduced the same mutation into the comparable mouse gene and assessed effects of the mutation on the metabolic state of the mice. We found the mutant mice recapitulated the hypertension phenotype and displayed abnormal fat distribution, but showed normal insulin sensitivity. A comparison of the phenotypes of Pparg ${ }^{P 465 L /+}$ mice with those of human patients $(6,11)$ and heterozygous PPAR $\gamma$ knockout $\left(\mathrm{Pparg}^{+/-}\right)$mice $(5,26,27)$ is summarized in Table 2.

The $\mathrm{P} 467 \mathrm{~L}$ mutation was originally identified in patients with severe insulin-resistant type 2 diabetes (11). Unlike human patients, however, $P$ parg ${ }^{P 465 L /+}$ mice maintained normal blood glucose and insulin levels and achieved better glucose homeostasis without significant changes in insulin sensitivity. Furthermore, the hyperinsulinemic-euglycemic clamp experiment in Pparg $g^{P 465 L /+}$ mice showed normal insulin sensitivity in peripheral tissues and liver, ruling out the possibility of insulin resistance. Although aging and diet have various effects on mice, 9-month-old (Table 1) and 12-month-old (data not shown) Pparg ${ }^{P 465 L /+}$ mice were no more insulin resistant than were wild-type mice, even when they were fed a high-fat diet for 6 months. Genetic background also plays an important role in the severity of insulin resistance in mice $(28,29)$. However, because the $F_{1}$ animals in our experiments were generated from two inbred lines, they are genetically identical except for proline or leucine at position 465 of PPAR $\gamma$. Consequently, any phenotypic differences between them are directly attributable to this substitution. Thus, the normal insulin sensitivity of Pparg ${ }^{P 465 L /+}$ mice demonstrates unequivocally that this mutation is not sufficient by itself to cause insulin resistance in mice.

This contrasts markedly with the severe insulin resistance observed in humans with the equivalent mutation. It is unlikely that the basic function of PPAR $\gamma$ differs between the two species. Conceivably, however, some undetermined modifier(s) of the phenotype may inherently differ in these two species. This possibility receives some support from the identification by Savage et al. of human patients with severe insulin resistance who are doubly heterozygous for frameshift mutations in one allele of the gene coding for PPAR $\gamma$ and for muscle-specific regulator subunit of protein phosphatase-1 (30). Both frameshifts cause premature stop and loss of function in the proteins, but individuals heterozygous for only one of these mutations are normal. This digenic inheritance suggests that a decreased level of PPAR $\gamma$ expression is insufficient by itself to cause insulin resistance, but it can do so when accompanied by a difference in the expression of a second gene.

The Pparg ${ }^{P 465 L /+}$ mice fed a high-fat diet had elevated insulin levels, accompanied by less-pronounced elevation of blood glucose, in response to acute glucose overload. The observed expansion of pancreatic islets in Pparg ${ }^{P 465 L /+}$ mice parallels the increased plasma insulin concentration and likely contributes to their better glucose responsiveness. Interestingly, the feature of elevated insulin levels, with occurrence of lower glucose curves during the IPGTT and normal insulin sensitivity, is similar to that seen in $U c p 2^{-/-}$mice (31) and pancreatic $\beta$ cell-specific overexpression of Akt1 (32). The functions of these gene products are known to be influenced by PPAR $\gamma$ agonists (33). The mechanism underlying the pancreatic islet function in Pparg ${ }^{P 465 L /+}$ mice requires further investigation.

Patients with the P467L mutation have partial lipodystrophy, as indicated by a loss of fat in subcutaneous depots in the limbs and buttocks but preserved visceral and abdominal subcutaneous fat (6). The lipodystrophy associated with PPAR $\gamma$ mutations in humans is variable, however. For example, an individual with an $\mathrm{R} 425 \mathrm{C}$ mutation in PPAR $\gamma$ has marked loss of subcutaneous fat from extremities with excess subcutaneous fat in the trunk (7). The F388L mutation in a three-generation Canadian kindred is associated with atrophy in gluteal fat and an accumulation of subcutaneous facial, suprascapular, and abdominal fat (8). Although our Pparg ${ }^{\mathrm{P} 65 \mathrm{LL} /+}$ mice demonstrate a causative link between the P465L mutation and body fat distribution, $P$ parg ${ }^{P 465 L /+}$ mice, unlike human patients, preferentially distribute fat toward extra-abdominal subcutaneous fat depots. Intra-abdominal adipose tissue has been suggested to play an important role in the pathogenesis of type 2 diabetes (34). The altered adipose tissue distribution away from visceral depots in Pparg ${ }^{P 465 L /+}$ mice may potentially prevent these mice from developing insulin resistance and may provide a possible explanation for the species discordance in metabolic sequelae. Intriguingly, the redistribution of body fat from the visceral to the subcutaneous depot in Pparg ${ }^{P 465 L /+}$ mice differs from the generalized reduction in fat tissue and body weight reported in Pparg - $^{+/}$mice (5) (Table 2) but is similar to that seen in humans or rodents treated with PPAR $\gamma$ agonists (35-37). The altered fat distribution in Pparg ${ }^{P 465 L /+}$ mice is likely to be a consequence of effects more complex than those caused by a 
simple reduction in PPAR $\gamma$ expression. For example, mutant PPAR $\gamma$ may sequester RXR $\alpha$ and other cofactors in a dominant negative fashion and exert effects different from those resulting from a simple reduction in PPAR $\gamma$.

Our results obtained with $P$ parg ${ }^{P 465 L /+}$ mice demonstrate that visceral fat and subcutaneous fat are biologically distinct and suggest that the roles of PPAR $\gamma$ may differ in different depots. Considerable evidence of marked differences in gene expression between visceral and subcutaneous fats also support the idea of intrinsic differences between visceral and subcutaneous adipose cells $(38,39)$. Given that the transcriptional activity of PPAR $\gamma$ is mediated by complex interactions with cofactors, heterodimeric RXR, and ligands, depot-specific differences in their availability likely influence the sensitivity to and dependency on PPAR $\gamma$ action in different adipose tissues. Consistent with this, the P465L mutation exerted depot-specific effects on gene expression. The selectively increased expression of LPL in inguinal adipose tissue promotes the delivery of FFA into the inguinal depot. The decreased expression of aP2 and Glut 4 in gonadal adipose tissue limits fuel uptake and storage by intra-abdominal fat depot. Combined alterations in PPAR $\gamma$ target genes may account for the partition of lipid preferentially to extra-abdominal subcutaneous fat rather than to the intra-abdominal fat depot in $P$ parg ${ }^{P 465 L /+}$ mice.

A majority of human diabetics suffer from both hyperglycemia and mild hypertension (40), and enhanced insulin sensitivity is associated with lower BP both in diabetic animals (41) and humans (19). The PPAR $\gamma$ agonist TZD has an antihypertensive effect that may result from a concurrent improvement in insulin sensitivity $(19,41)$. However, Pparg $^{P 465 L /+}$ mice are hypertensive from a young age without being insulin resistant, and the genotype effect on BP persists regardless of age or diet. Thus, PPAR $\gamma$ must have a role in $\mathrm{BP}$ regulation that is distinct from and independent of its role in the regulation of insulin sensitivity.

$\mathrm{BP}$ is ultimately determined by the filtration of blood in kidney glomeruli and the reabsorption of the filtrate in renal tubules. In the kidney, PPAR $\gamma$ is expressed mainly in the inner medulla (23), where sodium and water reabsorption takes place. However, our data indicate that the elevated BP of Pparg ${ }^{P 465 L /+}$ mice is not salt sensitive, suggesting that the role of PPAR $\gamma$ in the renal medulla on hypertension is not significant, at least in conditions we tested. No detectable increase in the systemic and kidney RAS is consistent with the absence of salt sensitivity. These results exclude the possibility of increased salt and water reabsorption in the kidney as a mechanism of hypertension in $\mathrm{Pparg}^{\mathrm{P} 465 \mathrm{~L} /{ }^{+}}$mice. Given that PPAR $\gamma$ is expressed in vasculature (20-22), further studies are necessary to determine the underlying mechanisms that affect BP in $\mathrm{Pparg}^{\mathrm{P465L/+}}$ mice, including potential involvement of PPAR $\gamma$ in the maintenance of vascular tone.

Adipose tissue is now recognized as an important endocrine organ that expresses genes encoding a large variety of bioactive molecules. Transgenic mice expressing AGT in adipose tissue have higher BP and increased fat mass (17). Our data show that the P465L mutation selectively increases expression of AGT in inguinal fat and of AT1R in gonadal fat, but does not affect expression of AGT in gonadal fat and of AT1R in inguinal fat. Angiotensin II (ATII) has been shown to act as a potent growth factor and plays a role in adipogenic growth and differentiation (15). The balance between the two ATII receptors, AT1R and AT2R, may influence adipocyte hypertrophy and adipose tissue hyperplasia (15). This raises the possibility that in the absence of proper adipogenesis mediated by PPAR $\gamma$, other default pathways, including the paracrine action of ATII, can help maintain differen- tiation of preadipocytes into mature adipocytes and/or hypertrophy of differentiated adipocytes. It is thus tempting to speculate that the increased RAS action in adipose tissues may in turn contribute to the elevated BP of Pparg ${ }^{P 465 L /+}$ mice by influencing the secretion of adipocytokines even if the adipose RAS does not directly affect the systemic RAS. Clearly, the mechanism underlying the differential regulations of AGT and AT1R in inguinal and gonadal adipose tissues in Pparg ${ }^{P 465 L /+}$ mice merits further studies.

In conclusion, our Pparg ${ }^{P 465 L /+}$ mice provide genetic evidence for an important link between PPAR $\gamma$ and the regulation of BP and fat distribution that is independent of any alteration in insulin sensitivity. Like human patients, $\operatorname{Pparg}^{P 465 L /+}$ mice are hypertensive. The mutation alters body fat storage in both human and mice, although the pattern of fat distribution in mice differs from that in humans. Finally, unlike human patients, $P$ parg ${ }^{P 465 L /+}$ mice are not insulin resistant. These similarities and differences in the phenotypes caused by an identical mutation in two species provide an opportunity to further dissect the role of PPAR $\gamma$ in the pathogenesis of the metabolic syndrome.

\section{Methods}

Generation of mice with the P465L mutation in PPAR $\gamma$. A missense mutation in the mouse Pparg gene that replaces proline (CCC) at position 465 in exon 6 with leucine (CTG) was produced by PCR-based site-directed mutagenesis. This fragment was cloned into the targeting vector, which contains a $6.5-\mathrm{kb} \mathrm{XhoI} / \mathrm{XbaI} 5^{\prime}$ homology fragment, the ACN (testis-specific Ace promoter, Cre recombinase, and Neo-resistance gene) cassette (42), a $1.3-\mathrm{kb}$ $\mathrm{XbaI} /$ StuI 3' homology fragment, and a thymidine kinase gene. Electroporation of the NotI-linearized vector into 129/SvEv ES cells (TC-1) and selection with G418 and ganciclovir were performed as described (43). ES cell clones with a successfully targeted Pparg locus were identified by PCR and confirmed by Southern blot analysis. Six male chimeras generated from targeted ES cells transmitted the mutated Pparg allele through the germline, and the lines were maintained on $129 / \mathrm{SvEv}$ genetic background. Studies were carried out using both male and female $\mathrm{F}_{1}$ offspring $(129 / \mathrm{SvEv}$ and C57BL/6) that were heterozygous and wild-type for PPAR $\gamma$. Mice were fed ad libitum either a regular chow diet (LabDiet 5P76; PMI Nutrition International, Richmond, Indiana, USA) or a high-fat diet (58\% of calories as fat and $26 \%$ as carbohydrate; D12330; Research Diets, New Brunswick, New Jersey, USA). Animals were handled following procedures approved by the Institutional Animal Care and Use Committees.

Culture of adipose tissue explants. Gonadal fat pads were removed from mice at 8 weeks of age ( $n=4$ each), cut into pieces $0.2-0.3 \mathrm{~cm}^{3}$ in size, and agitated for 30 minutes in DMEM (Cellgro, Herndon, Virginia, USA) supplemented with $1 \%$ BSA. Tissues were washed in PBS and were incubated at $37^{\circ} \mathrm{C}$ in six-well plates containing $3 \mathrm{ml}$ of DMEM supplemented with 10\% FBS (Atlanta Biologicals, Norcross, Georgia, USA), $100 \mathrm{IU} / \mathrm{ml}$ penicillin, $100 \mu \mathrm{g} / \mathrm{ml}$ streptomycin (Sigma-Aldrich, St. Louis, Missouri, USA), and various concentrations of rosiglitazone (Cayman Chemical, Ann Arbor, Michigan, USA) (44). After $6 \mathrm{~h}$, tissues were collected and stored in RNAlater (Ambion, Austin, Texas, USA) for RNA analysis.

RNA analysis. Tissue RNA from 8- to 10-week-old mice was extracted using the RNAeasy kit (Qiagen, Valencia, California, USA), and mRNA was analyzed with TaqMan (Applied Biosystems, Foster City, California, USA) real-time quantitative RT-PCR, with $\beta$-actin as reference gene in each reaction. 
Morphological analysis. Paraffin sections (6-10 $\mu \mathrm{m}$ in thickness) of adipose tissues isolated from female mice $(n=4$ each) at 10 weeks of age were cut and stained with H\&E. Adipocyte size was measured in 500 cells per mouse in several parts of the gonadal, inguinal, retroperitoneal, mesenteric, and pectoral fat pads using NIH Image. Pancreatic islet morphometry was analyzed using 14- to 16-week-old male mice fed either regular chow or a high-fat diet (D12330) for 4 weeks. Serial paraffin sections 5 $\mu \mathrm{m}$ in thickness from five different levels of pancreas $(100 \mu \mathrm{m}$ apart) were stained with H\&E. The mean islet area and endocrine mass were morphometrically determined by using four overlapping images (45).

IPGTT and IPITT. After mice had fasted for $5 \mathrm{~h}$, they were injected intraperitoneally with glucose $(1 \mathrm{~g} / \mathrm{kg}$ ) or insulin (Humulin; $0.5 \mathrm{U} /$ kg; Eli Lilly, Indianapolis, Indiana, USA). For the high-fat group, mice were fed a high-fat diet (D12330) for 4 or 22 weeks, beginning at 12 weeks of age. Blood was collected before and at indicated times after injections, as shown in Figures 3A and 3B. Plasma levels of glucose, total cholesterol, triglyceride, and FFA were determined using the appropriate reagent kits (Sigma-Aldrich). Insulin concentration was measured with rat insulin ELISAs (Crystal Chemical, Chicago, Illinois, USA). The insulin resistance index was calculated as the product of the areas under the glucose and insulin curves in the IPGTT as previously described (46).

Hyperinsulinemic-englycemic clamp. After an overnight fast, a 2-hour hyperinsulinemic-euglycemic clamp was established with primedcontinuous infusion of insulin (15 pmol $/ \mathrm{kg} / \mathrm{min}$; Humulin) and $\left[3-{ }^{3} \mathrm{H}\right]$ glucose $(0.1 \mu \mathrm{Ci} / \mathrm{min}$; NEN, Boston, Massachusetts, USA $)$ in 10 - to 12 -week-old male mice. Whole-body glucose turnover and glucose metabolic flux in Pparg ${ }^{P 465 L /+}$ mice $(n=5)$ and wild-type littermates $(n=5)$ were assessed as described previously (47). The effects of diet-induced alteration on insulin sensitivity were examined in $\mathrm{Pparg}^{\mathrm{P465L} / \mathrm{+}}$ mice $(n=8)$ and wild-type littermates $(n=5) \mathrm{fed}$ a high-fat diet (55\% of calories from fat and 7\% from sucrose; TD 93075; Harlan Teklad, Madison, Wisconsin, USA) for 3 weeks.

$B P$ measurements. BP and pulse rate were measured by the tailcuff method and were calculated as the average from three trials of five to ten measurements each day for 6 consecutive days (48). For continuous BP recording, a PA-C20 telemetric device (Data Sci- ences, St. Paul, Minnesota, USA) was implanted via the left carotid artery into the aortae of 24-week-old mice (49). Continuous BPs were recorded every 5 minutes for 14 days beginning 10 days after surgery to allow mice to regain normal diurnal rhythms.

Salt loading. Mice were fed a high-salt diet (8\% NaCl; TD 92012; Harlan Teklad) for 4 weeks, beginning at 10 weeks of age, and BP was measured by the tail-cuff method. Sodium balance during changes in dietary salt intake was assessed in mice housed in metabolic cages and fed a normal salt diet $(0.7 \% \mathrm{NaCl}$; LabDiet 5P76) for 7 days followed by a high-salt diet (8\% NaCl; TD 92012) for 7 days. Body weight, food and water intake, urine output, and urinary sodium excretion were measured each day (50).

Data analysis. Values are reported as mean \pm SEM. Statistical analysis was conducted using multifactorial ANOVA with genotype, gender, age, and diet as factors. Student's $t$ test was used for comparisons between mutant and wild-type within each group, and differences were considered to be statistically significant with $P$ values less than 0.05 .

\section{Acknowledgments}

We thank L. James, K. Caron, M. Kakoki, R. Coleman, J. Harp, K. Brown, C. Ammala, C.-J. Edgell, and O. Smithies for discussion and critical reading of the manuscript; R. Bagnell for assistance with microscopy and imaging; and S. Hiller, K. Kluckman, C. Wilhelm, L. Xu, E. Riggs, and A. Staton for technical assistance. The hyperinsulinemic-euglycemic clamp experiment was conducted at the NIH-Yale Mouse Metabolic Phenotyping Center. This work was supported by grants from the NIH (HL42630, HL70523 [N. Maeda], and DK 59635 [J.K. Kim]) the American Heart Association (0215270U [Y.S. Tsai]), and the American Diabetes Association (7-01-JF-05 [J.K. Kim]).

Received for publication January 5, 2004, and accepted in revised form May 20, 2004.

Address correspondence to: Nobuyo Maeda, 710 Brinkhous-Bullitt Building, University of North Carolina, Chapel Hill, North Carolina 27599, USA. Phone: (919) 966-6914; Fax: (919) 966-8800; E-mail: nobuyo@med.unc.edu.
1. Reaven, G.M. 1988. Role of insulin resistance in human disease. Diabetes. 37:1595-1607.

2. Berger, J., and Moller, D.E. 2002. The mechanisms of action of PPARs. Annu. Rev. Med. 53:409-435.

3. Tontonoz, P., Hu, E., and Spiegelman, B.M. 1994. Stimulation of adipogenesis in fibroblasts by PPAR gamma 2, a lipid-activated transcription factor. Cell. 79:1147-1156.

4. Barak, Y., et al. 1999. PPAR gamma is required for placental, cardiac, and adipose tissue development. Mol. Cell. 4:585-595.

5. Kubota, N., et al. 1999. PPAR gamma mediates high-fat diet-induced adipocyte hypertrophy and insulin resistance. Mol. Cell. 4:597-609.

6. Savage, D.B., et al. 2003. Human metabolic syndrome resulting from dominant-negative mutations in the nuclear receptor peroxisome proliferator-activated receptor-gamma. Diabetes. 52:910-917.

7. Agarwal, A.K., and Garg, A. 2002. A novel heterozygous mutation in peroxisome proliferatoractivated receptor-gamma gene in a patient with familial partial lipodystrophy. J. Clin. Endocrinol. Metab. 87:408-411.

8. Hegele, R.A., Cao, H., Frankowski, C., Mathews, S.T., and Leff, T. 2002. PPARG F388L, a transactivationdeficient mutant, in familial partial lipodystrophy.
Diabetes. 51:3586-3590.

9. Deeb, S.S., et al. 1998. A Pro12Ala substitution in PPARgamma2 associated with decreased receptor activity, lower body mass index and improved insulin sensitivity. Nat. Genet. 20:284-287.

10. Ristow, M., Muller-Wieland, D., Pfeiffer, A., Krone, W., and Kahn, C.R. 1998. Obesity associated with a mutation in a genetic regulator of adipocyte differentiation. N. Engl. J. Med. 339:953-959.

11. Barroso, I., et al. 1999. Dominant negative mutations in human PPARgamma associated with severe insulin resistance, diabetes mellitus and hypertension. Nature. 402:880-883.

12. Stamler, R., Stamler, J., Riedlinger, W.F., Algera, G., and Roberts, R.H. 1978. Weight and blood pressure. Findings in hypertension screening of 1 million Americans. JAMA. 240:1607-1610.

13. Bonora, E., et al. 1998. Prevalence of insulin resistance in metabolic disorders: the Bruneck Study. Diabetes. 47:1643-1649.

14. Ginsberg, H.N. 2000. Insulin resistance and cardiovascular disease. J. Clin. Invest. 106:453-458.

15. Engeli, S., Negrel, R., and Sharma, A.M. 2000. Physiology and pathophysiology of the adipose tissue renin-angiotensin system. Hypertension. 35:1270-1277.
16. Hainault, I., et al. 2002. Adipose tissue-specific increase in angiotensinogen expression and secretion in the obese (fa/fa) Zucker rat. Am. J. Physiol. Endocrinol. Metab. 282:E59-E66.

17. Massiera, F., et al. 2001. Adipose angiotensinogen is involved in adipose tissue growth and blood pressure regulation. FASEB J. 15:2727-2729.

18. Dubey, R.K., Zhang, H.Y., Reddy, S.R., Boegehold, M.A., and Kotchen, T.A. 1993. Pioglitazone attenuates hypertension and inhibits growth of renal arteriolar smooth muscle in rats. Am. J. Physiol. 265:R726-R732.

19. Ogihara, T., Rakugi, H., Ikegami, H., Mikami, H., and Masuo, K. 1995. Enhancement of insulin sensitivity by troglitazone lowers blood pressure in diabetic hypertensives. Am. J. Hypertens. 8:316-320.

20. Marx, N., Schonbeck, U., Lazar, M.A., Libby, P., and Plutzky, J. 1998. Peroxisome proliferator-activated receptor gamma activators inhibit gene expression and migration in human vascular smooth muscle cells. Circ. Res. 83:1097-1103.

21. Delerive, P., et al. 1999. Peroxisome proliferatoractivated receptor activators inhibit thrombininduced endothelin-1 production in human vascular endothelial cells by inhibiting the activator protein-1 signaling pathway. Circ. Res. 85:394-402. 
22. Marx, N., Bourcier, T., Sukhova, G.K., Libby, P., and Plutzky, J. 1999. PPARgamma activation in human endothelial cells increases plasminogen activator inhibitor type-1 expression: PPARgamma as a potential mediator in vascular disease. Arterioscler. Thromb. Vasc. Biol. 19:546-551.

23. Yang, T., et al. 1999. Expression of peroxisomal proliferator-activated receptors and retinoid $\mathrm{X}$ receptors in the kidney. Am. J. Physiol. 277:F966-F973.

24. Takeda, K., et al. 2000. Peroxisome proliferatoractivated receptor gamma activators downregulate angiotensin II type 1 receptor in vascular smooth muscle cells. Circulation. 102:1834-1839.

25. Matsubara, H. 1998. Pathophysiological role of angiotensin II type 2 receptor in cardiovascular and renal diseases. Circ. Res. 83:1182-1191.

26. Miles, P.D., Barak, Y., He, W., Evans, R.M., and Olefsky, J.M. 2000. Improved insulin-sensitivity in mice heterozygous for PPAR-gamma deficiency. J. Clin. Invest. 105:287-292.

27. Asakawa, M., et al. 2002. Peroxisome proliferatoractivated receptor gamma plays a critical role in inhibition of cardiac hypertrophy in vitro and in vivo. Circulation. 105:1240-1246.

28. Kulkarni, R.N., et al. 2003. Impact of genetic background on development of hyperinsulinemia and diabetes in insulin receptor/insulin receptor substrate-1 double heterozygous mice. Diabetes. 52:1528-1534.

29. Kido, Y., Philippe, N., Schaffer, A.A., and Accili, D. 2000. Genetic modifiers of the insulin resistance phenotype in mice. Diabetes. 49:589-596.

30. Savage, D.B., et al. 2002. Digenic inheritance of severe insulin resistance in a human pedigree. Nat. Genet. 31:379-384.

31. Zhang, C.Y., et al. 2001. Uncoupling protein-2 negatively regulates insulin secretion and is a major link between obesity, beta cell dysfunction, and type 2 diabetes. Cell. 105:745-755.

32. Tuttle, R.L., et al. 2001. Regulation of pancreatic beta-cell growth and survival by the serine/threonine protein kinase Akt1/PKBalpha. Nat. Med. 7:1133-1137.

33. Medvedev, A.V., Snedden, S.K., Raimbault, S., Ricquier, D., and Collins, S. 2001. Transcriptional regulation of the mouse uncoupling protein-2 gene. Double E-box motif is required for peroxisome proliferator-activated receptor-gamma-dependent activation. J. Biol. Chem. 276:10817-10823.

34. Montague, C.T., and O'Rahilly, S. 2000. The perils of portliness: causes and consequences of visceral adiposity. Diabetes. 49:883-888.

35. Kelly, I.E., Han, T.S., Walsh, K., and Lean, M.E. 1999. Effects of a thiazolidinedione compound on body fat and fat distribution of patients with type 2 diabetes. Diabetes Care. 22:288-293.

36. Arioglu, E., et al. 2000. Efficacy and safety of troglitazone in the treatment of lipodystrophy syndromes. Ann. Intern. Med. 133:263-274.

37. Laplante, M., et al. 2003. PPAR-gamma activation mediates adipose depot-specific effects on gene expression and lipoprotein lipase activity: mechanisms for modulation of postprandial lipemia and differential adipose accretion. Diabetes. 52:291-299.

38. Montague, C.T., et al. 1998. Depot-related gene expression in human subcutaneous and omental adipocytes. Diabetes. 47:1384-1391.

39. Atzmon, G., et al. 2002. Differential gene expression between visceral and subcutaneous fat depots. Horm. Metab. Res. 34:622-628.

40. Ferrari, P., and Weidmann, P. 1990. Insulin, insulin sensitivity and hypertension. J. Hypertens. 8:491-500.

41. Walker, A.B., Chattington, P.D., Buckingham, R.E., and Williams, G. 1999. The thiazolidinedione rosiglitazone (BRL-49653) lowers blood pressure and protects against impairment of endothelial function in Zucker fatty rats. Diabetes. 48:1448-1453.

42. Bunting, M., Bernstein, K.E., Greer, J.M., Capecchi, M.R., and Thomas, K.R. 1999. Targeting genes for self-excision in the germ line. Genes Dev. 13:1524-1528.

43. Piedrahita, J.A., Zhang, S.H., Hagaman, J.R., Oliver, P.M., and Maeda, N. 1992. Generation of mice carrying a mutant apolipoprotein $\mathrm{E}$ gene inactivated by gene targeting in embryonic stem cells. Proc. Natl. Acad. Sci. U. S. A. 89:4471-4475.

44. Glorian, M., et al. 2001. A single element in the phosphoenolpyruvate carboxykinase gene mediates thiazolidinedione action specifically in adipocytes. Biochimie. 83:933-943.

45. Pende, M., et al. 2000. Hypoinsulinaemia, glucose intolerance and diminished beta-cell size in S6K1deficient mice. Nature. 408:994-997.

46. Yamauchi, T., et al. 2001. The fat-derived hormone adiponectin reverses insulin resistance associated with both lipoatrophy and obesity. Nat. Med. 7:941-946.

47. Kim, J.K., et al. 2000. Redistribution of substrates to adipose tissue promotes obesity in mice with selective insulin resistance in muscle. J. Clin. Invest. 105:1791-1797.

48. Krege, J.H., Hodgin, J.B., Hagaman, J.R., and Smithies, O. 1995. A noninvasive computerized tail-cuff system for measuring blood pressure in mice. Hypertension. 25:1111-1115.

49. Butz, G.M., and Davisson, R.L. 2001. Long-term telemetric measurement of cardiovascular parameters in awake mice: a physiological genomics tool. Physiol. Genomics. 5:89-97.

50. Oliverio, M.I., Best, C.F., Smithies, O., and Coffman, T.M. 2000. Regulation of sodium balance and blood pressure by the $\mathrm{AT}(1 \mathrm{~A})$ receptor for angiotensin II. Hypertension. 35:550-554. 